\title{
Cerebral Malaria in Children : An Update
}

\author{
M M Z Islam ' , M M Rahman²
}

\begin{abstract}
Cerebral malaria (CM) is the most severe neurological presentation of acute falciparum malaria. It is a medical emergency, the hallmark of which is the presence of coma probably due to diffuse encephalopathy. A compromised microcirculation with sequestration of parasitized erythrocytes is central to the pathogenesis of cerebral malaria. The death is unacceptably high even with effective antimalarials in tertiary care hospital. The mainstay of treatment of cerebral malaria include prompt diagnosis and early institution of effective antimalarial therapy, recognition of complications, and appropriate supportive management in an ICU. Neurological sequlae are increasingly recognized, but further research on the pathogenesis of coma and neurological damage is required to develop other ancillary treatments.
\end{abstract}

Key words : Cerebral malaria, encephalopathy, antimalarials.

\section{Introduction}

Malaria is one of the most important of the parasitic diseases of humans, and its neurological complications due to cerebral malaria is arguably one of the most common non-traumatic encephalopathies in the world. ${ }^{1}$ Malaria remains a major public health problem in most tropical countries. It has been estimated that 300 to 500 million individuals are affected annually and about 1.5 to 2.5 million people die of malaria every year in spite of decades of extensive research, an effective vaccine against this deadly disease is still out of reach. ${ }^{2}$

${ }^{1}$ Dr. Mirza Md. Ziaul Islam Assistant Professor Dept. of Pediatric Infectious Diseases \& Community Pediatrics Dhaka Shishu (Children) Hospital

${ }^{2}$ Dr. M Mizanur Rahman Associate professor \& Head, Dept. of Pediatric Infectious Diseases \& Community Pediatrics Dhaka Shishu (Children) Hospital

Correspondence

Dr. Mirza Md. Ziaul Islam, Assistant Professor Dept. of Pediatric Infectious Disease \& Community Pediatrics Dhaka Shishu (Children) Hospital Sher-e-Bangla Nagar, Dhaka e-mail: mirzamd. ziaulislam @yahoo.com
Five species of plasmodium can cause malaria in man, namely, P. vivax, P. falciparum, P. ovale, P. malaria and P. knowlesi. ${ }^{3}$ Plasmodium falciparum is responsible for almost all the mortality from malaria and is the only species that appear to directly affect the central nervous system causing neurological deficits and cognitive sequlae. ${ }^{4}$ Falciparum malaria causes more than one million deaths each year and also contributes significantly to many other deaths,specially in young children. ${ }^{5}$ Severe malaria is a potentially fatal multisystem disease of which cerebral malaria, characterized by unrousable coma, is one of the most common features. ${ }^{6}$ Cerebral malaria is a medical emergency demanding urgent clinical assessment and treatment. ${ }^{1}$ It has been observed that most of the severe malarial deaths, mainly cerebral type, occur in the first 24 hours of hospitalization, in spite of effective antimalarials (quinine or artemisinine) ${ }^{2}$. Hence, in order to decrease the mortality from cerebral malaria, we need to have a clear conception about the case definitions, clinical features, pathophysiology diagnosis and the adjuvant therapies along with antimalarials.

\section{Definition}

Cerebral malaria is defined as a deep level of unconsciousness (inability to localise a painful stimulus) in the presence of a P. faciparum asexual parasitaemia, after the correction of hypoglycemia and exclusion of other encephalopathies, especially bacterial meningitis and locally prevalent viral encephalitis. ${ }^{7,8}$

\section{Pathophysiology}

The histopathological hallmark of cerebral malaria is the engorgement of cerebral capillaries and venules with parasitized red blood cells $\left(\mathrm{PRBC}_{\mathrm{s}}\right.$ ) and the nonparasitized red blood cells $\left.\left(\mathrm{NPRBC}_{\mathrm{s}}\right)\right)^{9}$ The brain is usually swollen at postmortem and immunohistochemical staining suggests endothelial activation and disruption of blood brain barrier. ${ }^{10,11}$ The sequestration of red cells containing mature forms of the parasite (trophozoites and schizonts) in the microvasculature is thought to cause the major complications of falciparum malaria, particularly cerebral malaria. ${ }^{12}$ It is the sequestered parasites that cause pathology in severe malaria, and prognosis is related to the sequestered biomass. ${ }^{13}$ Sequestration is thought to be a specific interaction between PRBCs and vascular endothelium (coadherence) which reduces the microvascular blood flow and is thought to be the cause of organ and tissue dysfunction. ${ }^{14}$

\section{Clinical features}

Cerebral malaria is one of the features of severe malaria which is a multisystem disease. ${ }^{15}$ The three consistent presenting complaints of cerebral malaria (CM) are fever, convulsion and loss of consciousness. ${ }^{16}$ The clinical picture of cerebral malaria is that of a diffuse encephalopathy with unrousable coma; focal signs are relatively uncommon. In young children coma can develop rapidly, with a mean onset after only 2 days of fever, but sometimes just a few hours. ${ }^{17}$ It is often heralded by one or more generalized seizures, which cannot be distinguished clinically from febrile convulsions. In more than $50 \%$ of pediatric cases have convulsions which are most frequently generalized tonic-clonic in nature but can also be Jacksonian type or focal. In small children, approximately $25 \%$ subtle or 
subclinical convulsions with seizure activity on EEG, but only minor convulsive movements of limbs or facial musceles. ${ }^{17,18}$ These patients often have deviated eyes, upward rolling of eyes, excessive salivation and irregular breathing pattern. ${ }^{17}$

On neurological examination, the febrile patient has no signs of meningism, although passive resistance to neck flexion is not uncommon and hyperextension of the neck may occur. ${ }^{17}$ The eyes often show a divergent gaze, with normal oculocephalic reflexes. Pupillary and corneal reflexes are usually normal but may be absent in deeply comatose patient. ${ }^{17,19,20}$ On fundoscopy, retinal hemorrhages can be observed in about $15 \%$ of cases. These hemorrhages are boat or flame shaped and they seldom invade macula. 17,20

Cranial nerve involvement in patients with $\mathrm{CM}$ is rare. Muscle tone and tendon reflexes are often increased, but can also be normal or reduced. An ankle reflex, frequently a patellar clonus can sometimes be evoked. Abdominal reflexes are absent and the planter reflexes are extensor in approximately half of the cases. Various forms of abnormal posturing can be present, with either a decorticate pattern with flexor rigidity or a decerebrate pattern with abnormal extensor responses in arms and legs with or without ophistotonus. ${ }^{17,18}$

\section{Diagnosis}

In the tropical countries, cerebral malaria should be considered in early comatose patient with a history of fever who has been in a malarious area in the previous two months.

In clinical practice, the diagnosis of cerebral malaria is based on findings asexual form of $P$. falciaprum in the peripheral blood of a febrile patient with unrousable coma lasting more than 30 minutes after seizures had stopped, for which no other cause was found by clinical and laboratory tests. ${ }^{21}$

Microscopic examination of a blood film remains the gold standard for diagnosis of malaria. ${ }^{22}$ Both thick and thin film should be examined. Thick film have a higher sensitivity for diagnosis while thin films allow more accurate speciation and quantification of parasitaemia. ${ }^{23}$ Rapid diagnostic tests (RDTs) are used in many settings. RDTs detect circulating parasite associated proteins and enzymes. Most tests detect both a pan-species target and a falciparum-specific target. ${ }^{22}$ Lumbar puncture (LP) to be done for analysis of cerebrospinal fluid (CSF) to exclude bacterial meningitis which shares some features with cerebral malaria. ${ }^{17}$ Other investigations include full blood count, blood glucose, liver function test, kidney function test, blood culture (s) for typhoid, bacteremia or both, chest-x-ray to exclude community acquired pneumonia. $^{7}$

\section{Management}

Cerebral malaria is a medical emergency demanding urgent clinical assessment and treatment. ${ }^{1}$ The main objective of treatment is to prevent the mortality and the secondary objectives are prevention of disabilities and prevention of recrudescence. ${ }^{2}$ The mortality of untreated cerebral malaria is thought to approach $100 \%$. With prompt, effective antimalarial treatment and supportive care, the mortality falls to 15$20 \%$ overall. $^{25}$

The mainstay of the treatment of cerebral malaria is the commencement of parenteral antimalarial treatment. Available drugs are injectable artesunate, quinine and artemether. IV chloroquine has become obsolete in Asia and almost the whole rest of the world because of widespread resistence of the parasite to this. ${ }^{6}$ Artesunate belongs to the group of artemisinins, which are currently the most rapidly acting and potent antimalarial drugs. Unlike quinine, they can act both on the mature and immature form of the parasites. Treatment with artesunate is well tolerated, whereas quinine is associated with hypoglycaemia. The dose of artesunate is $2.4 \mathrm{mg} / \mathrm{kg}$ body weight on admission, followed by the same dose after 12 and 48 hours, and then daily until the patient is able to take oral medication.

To prevent recrudescence of the infection, follow on medication should be given. Several regimens are possible, such as a full course of artemether-lumefantrine (co-artem ${ }^{R}$ ) (total course7days including the parenteral form) and clindamycin (10mg/kg bd. for 7 days). ${ }^{26,27}$

Until parenteral artesunate becomes more widely registered and available in more countries, most patients in Asia will currently still be treated with parenteral quinine. A loading dose of $20 \mathrm{mg} / \mathrm{kg}$ body weight over 4 hours should be given by controlled infusion with dextrose (caution : can lead to fatal hypotension). The loading dose is followed by a dose of $30 \mathrm{mg} / \mathrm{kg} / 24$ hours as a continuous infusion or in 3 divided doses of $10 \mathrm{mg} / \mathrm{kg}$ each given over 4 hours time. The total course is seven days of quinine. Quinine can be switched to oral formulation, using the same dose of $10 \mathrm{mg} / \mathrm{kg}$ t.i.d., if the patient is able to eat and this should be combined with clindamycin. ${ }^{21}$

Unfortunately, the treatment of cerebral malaria does not end with the clearance of the parasites. The clinical picture of cerebral malaria may persist or even become worse with passage of time inspite of the clearance of parasites from blood. Moreover, complications like acute renal failure, acute lung injury, etc. may set in. As no definitive drug is available to combat these complications, supportive symptomatic therapy are required. It has been observed that most of the malarial deaths occur in the first 24 hours of hospitalization, inspite of effective antimalarials. Hence, there is an urgent need of management of multiple complications along with definitive antimalarial treatment. ${ }^{2}$

An open airway should be secured in unconscious patients and breathing and circulations established. Immediate correction of hypoglycemia (if present) to be corrected by I/V dextrose. The degree of acidosis is an important determinant of outcome; the plasma bicarbonate or venous lactate level should, therefore, be measured including arterial $\mathrm{p}^{\mathrm{H}}$ and steps to be taken to correct. The assessment of fluid balance is critical in cerebral malaria. Respiratory distress, in particular with acidotic breathing in severely anemic children, often indicate hypovolemia and requires prompt rehydration, and, when indicated, blood transfusion. Higher doses of phenobarbital are needed to prevent convulsions in children. If there is evidence of cerebral swelling, 20\% mannitol solution should be infused. In some patients, broad spectrum antibiotics are needed as concomitant septicaemia is not uncommon. ${ }^{28}$

\section{Outcome}

Death among hospitalized children with cerebral malaria is often due to respiratory arrest and brain stem signs and most deaths occur within 24 hours of presentation in hospital. ${ }^{29}$ In surviving patients, the median time to full recovery of consciousness is approximately 24 hours in children, compared to 48 hours in adults. ${ }^{30}$ During discharge neurological residual abnormalities(including hemiplegia, cortical blindness, aphasia and cerebellar araxia etc.) are more common in 
children, approximately $12 \% .^{31,32}$ The prevalence of neurological deficits of cerebral malaria ranges between 9 and $17.7 \% .^{31,32,33}$ These symptoms often resolve completely over a period from one to six months in over half of the children, but a quarter will be left with major neurological deficits. ${ }^{31,32}$

\section{Conclusion}

Cerebral malaria is a life threatening complication of malaria. It affects children more than adult and should be considered in any patient with impairment of consciousness. The mortality rate is high and a significant number of childhood survivors suffer from transient neurological deficit at discharge and subtle long-term cognitive deficiencies. High index of suspicision is needed for early diagnosis and effective treatment. Urgent treatment with antimalarial drug is required, but the prognosis often depends on the management of complications.

\section{References}

1. Charles RJC, Hien T, White N. Cerebral Malaria. J Neurol P Neurosurg Psychiatry 2000;69:433-44.

2. Mahanty S, Patel DK, Pati SS, Mishra SS, et al. Adjunct therapy in cerebral malaria. Indian J Med Res 2006; 124:245-260.

3. Singh B, Kim Sung L, Shamsul SS. A large focus of naturally acquired plasmodium knowlesi in infections in human beings. Lancet 2004;363:1017-24.

4. Newton CR, Hien TT, White N. Cerebral malaria. J NeurolNeurosurg Psychiatry 2000; 69:433-41.

5. WHO (2005) Global Malaria Situation. World Malaria Report 2005. WHO/HTM/MAL/ 2005,1102, Geneva: RBM/UNICEF/World Health Organization 5-17.

6. Arjen M Dondor P. Neurology Asia 2005;10:67-77.

7. Warell DA, Molyneux ME, Beales PF. Severe and complicated malaria. Trans R Soc Trop Med Hyg 1990: 84 (suppl 2):1-65.

8. World health Organization. Management of severe malaria: a practical handbook, $2^{\text {nd }}$ ed. Geneva World Health Organization,2000.

9. Mac Pherson GG. Warell MJ, White NJ, et al. Human cerebral malaria. A quantitative ultrastructural analysis of parasitized erythrocyte sequestration. AM J Pathol 1985; 119:385-401.

10. Turner $\mathrm{G}$ DH, Morrison $\mathrm{H}$, Jones M. An immunohistochemical study of the pathology of fatal malaria. Am J Pathol1994; 145:1057- 69.

11. Brown $\mathrm{H}$, Hien $\mathrm{T}$, Day N. Evidence of blood brain barrier dysfunction in human cerebral malaria. NeuropatholAppl Neurobiol 1999;25:331-40.

12. White NJ, Ho M. The Pathophysiology of malaria. AdvParasitol 1992;3:83-173.

13. Davis TME, Krishna $S$, Looareesuwan S. Erythrocyte sequestration and anemia in severe falciparum malaria. J Clin Invest 1990;86:793-800.
14. KaulDk, Liu XD, Nagel RL. Microvascular hemodynamics and in vivo evidence for the role of intercellular adhesion molecule- 1 in the sequestration of infected red blood cells in a mouse model of lethal malaria. Am J Trop Med Hyg 1998;58:240-247.

15. Marsh $\mathrm{K}$, Forster $\mathrm{D}$, Waraira $\mathrm{C}$. Indicators of life threatening malaria in African Children. N Engl Med 1995;332:1399-1404.

16. Oluwayemi OI, Brown BJ, Oysdeji OA, et al. Clinical and laboratory predictors of outcome in cerebral malaria in suburban Nigeria. J Infect Dev Ctries 2013;7:600-607.

17. World Health Organization Communicable Diseases Cluster, Severe falciparum malaria. Trans R Soc Trop Med Hyg 2000;94 (s-1):1-90.

18. Kakkihya BS. History of Malaria. Malria site 2014.

19. Olumese PE, Gbodegesin RA, AdeymoAA, Brown B, et al. Neurological features of cerebral malaria in Nigerian children, Am Trop Pediatr 1999; 19:321-325.

20. Lewallen S, Haeding SP, Ajewole. A review of the spectrum of clinical, ocular fundus findings in P.falciparum malaria in Africa children with a proposed classification and grading system. Trans T Soc Trop Med Hyg 1999;93:619-22.

21. WHO Guidelines for the treatment of Malaria 2010. Switzerland: World Health Organization.

22. Lallo DG, Shingadia D, Pasval G. UK malaria treatment guidelines. J Infect 2007;54:111121

23. Payne D. Use and limitations of light microscopy for diagnosis of malaria. Bull World Health Organ 1988; 66: 621-6.

24. World Health Organization. Guidelines for the treatment of Malaria, $2^{\text {nd }}$ Edn. Geneva, 2011.

25. Marks M, Gupta Wright A, Doherty JF, Singe M, Walker D, et al. Managing Malaria in the intensive care unit. British Journal of Anaesthesia 2014. doi:10.1093/bja/aeu,57.

26. Ajayi IO. Feasibility and acceptability of artemisinin-based combination therapy for the management of malaria in four African Sites. Malaria Journal 2008;7:6.doi.1186/14752875 .

27. Artesunatevs.quinine in the treatment of severe falciparum malaria in African children (AQUAMT): an open-label randomized trial. Lancet 2010;376:1647-57.

28. World Health Organization, Severe falciparum malaria. Transaction of the Royal Society of Tropical Medicine and Hygiene 2000;94 (Suppl-1):1-90.

29. Looareesuwan S, Warell DA, White NJ. Retional hemorrhage, a common sign of prognostic significance in cerebral malaria. Am J Trop Med Hyg 1983;32:911-915.

30. Molyneux ME, Taylor TE, Wirima JJ. Clinical features and prognostic indicators in pediatric cerebral malaria: a study of 131 comatosmalarian children. QJ Med 1989; 71:441-459.

31. Oluwayemi IO, Brown BJ, Oyedeji OA. Neurological sequlae in survivors of cerebral malaria. Pan Afr Med J 2013:15:88.

32. Idro R, Marash K, John CC. Cerebral malaria: Inechanisms of brain injury and strategies for improved neurocognitive outcome, Pediatr Res 2010;68:287-274.

33. Holding PA, Stevenson J, Peshu N, Marsh K. Cognitive sequlae of severe malaria with impaired consciousness. Trans R soc Trop Med Hyg 1999;93:529-534. 